\title{
An improved waveform for multi-target detection in FMCW vehicle radar
}

\author{
Jian $\mathrm{Li}^{1, \mathrm{a}}$, Wenquan Che ${ }^{1, \mathrm{~b}}$, Tao Shen ${ }^{1, \mathrm{c}}$, Wenjie Feng ${ }^{1, \mathrm{~d}}$, Xuyang $\mathrm{Li}^{1, \mathrm{e}}$ and \\ Kuan Deng ${ }^{2, f}$ \\ ${ }^{1}$ School of Electronic and Optical Engineering, Nanjing University of Science and Technology, \\ Nanjing 210094, China; \\ ${ }^{2}$ School of Electronic Information Engineering, Jinling Institute of Technology, \\ Nanjing 211169, China. \\ alijian918439@163.com, byeeren_che@163.com, 'shentao199212@163.com
}

Keywords: FMCW, multi-target detection, radar waveform

\begin{abstract}
Frequency Modulation Continuous Wave (FMCW) radar has been widely used in vehicle safety systems now. Many kinds of waveform have been designed to solve the coupling of distance and velocity in multi-target detection. This paper proposes a novel FMCW waveform which contains three sections with same duration in each period. Two sections are up ramps with different slopes and one is down ramp. Two groups of $\mathrm{N}^{*} \mathrm{~N}$ values of the distance and velocity can be extracted by implementing N-point FFT towards one up ramp and down ramp signal. The distance and velocity in the two groups are the same for the real targets, however, quite different for the ghost targets. Compared with traditional waveforms, the proposed waveform in our work has simpler algorithm and less computation, which has been validated by simulations.
\end{abstract}

\section{Introduction}

Compared with the general radar, LFMCW radar has a large time-bandwidth product, therefore it has high ranging precision and resolution. In addition, it also takes the features of simple structure, small volume, light weight, high immunity to weather and environment, and thus has been widely applied in automotive anti-collision radar, automatic cruise control [1].

Because of the relative motion between radar and target, there is the coupling problem of distance and velocity. The traditional radar transmits the symmetric triangular wave signal, and the distance and velocity parameters of the target are obtained by using the symmetry of the up and down beat signals. But when there are multiple targets, it is difficult to obtain the accurate parameters of the targets because of the false targets. To solve such problems, [2] presented a changeable periods frequency modulated continuous wave radar signal, while [3] proposed a changeable slope continuous wave radar signal .And [4]-[6] also proposed some other waveforms. These methods can solve the multi-target detection problem in eliminating false targets, but they have the problem of large amount of computation and complex algorithm, which will lead to increased cost and poorer performance of the vehicle borne radar.

In this work, a new radar waveform is proposed, which is easy to implement in practice and only need to implement FFT three times.

\section{Multi-target detection of FMCW Radar}

\subsection{Principle of FMCW radar}

The basic principle of FMCW radar is to determine the speed and distance of the target by the frequency difference between the transmitting signal and the receiving signal, and the traditional FMCW signal is shown in Fig. 1 , where $f_{0}$ is the starting frequency of the modulation signal, $B$ is the modulation bandwidth, and $T$ is the modulation period. Noting that, $f_{d}$ is the Doppler frequency, which can be expressed as $f_{d}=\frac{2 v}{\lambda} \cdot f_{\tau}$ is the beat frequency of stationary target, expressed 
as $f_{\tau}=\frac{4 R B}{C T} \cdot f_{+}$refers to the up ramps beat frequency and correspondently $f_{-}$is the down ramps beat frequency which can be expressed as follows:

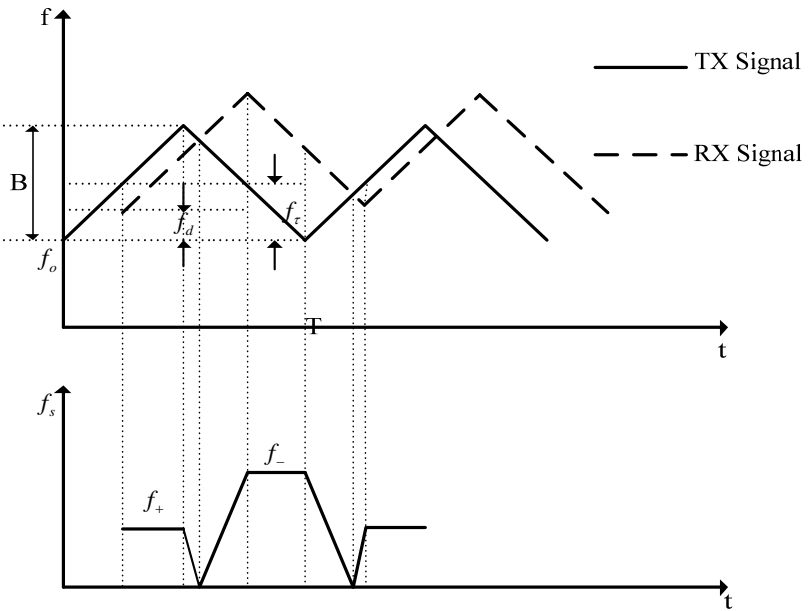

Fig. 1 Principle of FMCW radar for a single moving target using triangular waveform

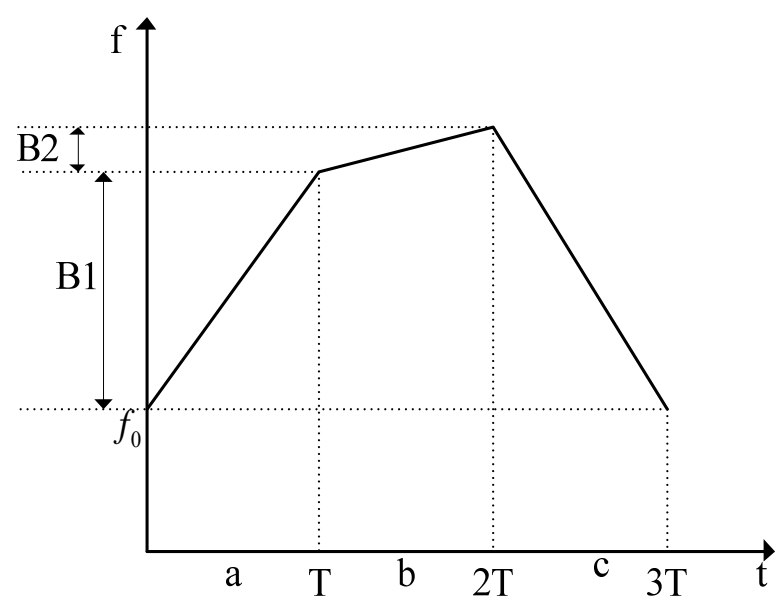

Fig. 2 The proposed radar waveform

$$
f_{+}=f_{\tau}-f_{d} \quad f_{-}=f_{\tau}+f_{d}
$$

Therefore, the distance $\mathrm{R}$ and the relative velocity $\mathrm{v}$ of the target can be extracted as follows:

$$
\begin{aligned}
& R=\frac{c T}{8 B}\left(f_{+}+f_{-}\right) \\
& v=\frac{c}{4 f_{0}}\left(f_{-}-f_{+}\right)
\end{aligned}
$$

In the case of detecting a single target, this method is simple and feasible. While in a multi-target situation ( $N$ targets is assumed), there are $\mathrm{N}$ beat frequencies in the up ramp $\left(f_{a 1}, f_{a 2}, \ldots, f_{a n}\right)$ and $N$ beat frequencies in the down $\operatorname{ramp}\left(f_{b 1}, f_{b 2}, \ldots, f_{b n}\right)$, if the possibility of the combination of these two groups of lines is considered, the $\mathrm{N}^{2}$ groups of distance and velocity can be obtained. However, only $\mathrm{N}$ out of $\mathrm{N}^{2}$ are real targets and the rest are so-called ghost targets.

\subsection{The proposed method}

To solve above problem, a new radar waveform is proposed in this work, as shown in Figure 2. Two segments are up ramps with different slopes $\mu_{1}=\frac{B_{1}}{T}, \mu_{2}=\frac{B 2}{T}$ and one segment is down ramp with the slopes $\mu_{3}=\frac{B_{3}}{T}$. According to the matching algorithm, the true distance and velocity of targets can be obtained accurately. It can not only solve the problem of how to eliminate false targets in target detection, but also only need a simple algorithm which can reduce the amount of computation effectively.

For section $a, b$ and $c$, the following expressions can be obtained from (1)

$$
f_{a+}=f_{a \tau}-f_{a d} \quad f_{b+}=f_{b \tau}-f_{b d} \quad f_{c-}=f_{c \tau}+f_{c d}
$$

The value of the distance and velocity are obtained by section $a$ and $c$,

$$
R_{1}=\frac{c\left(f_{a+}+f_{c-}\right)}{2\left(\mu_{1}+\mu_{3}\right)} \quad v_{1}=\frac{c\left(\mu_{3} f_{a+}-\mu_{1} f_{c-}\right)}{2 f_{0}\left(\mu_{1}+\mu_{3}\right)}
$$

In the same way, the value of the distance and velocity are obtained by section $b$ and $c$.

$$
R_{2}=\frac{c\left(f_{b+}+f_{c-}\right)}{2\left(\mu_{2}+\mu_{3}\right)} \quad v_{2}=\frac{c\left(\mu_{3} f_{b+}-\mu_{2} f_{c-}\right)}{2 f_{0}\left(\mu_{2}+\mu_{3}\right)}
$$


In the signal processing, 1024-point FFT of section $a, b$ and $c$ are implemented respectively. According to (5) 6) $N^{*} N$ groups of value of the distance and velocity are obtained by section $a$ and section $c$, then $\mathrm{N}^{*} \mathrm{~N}$ groups of value of the distance and velocity are obtained by section $b$ and section $c$. Generally, the two group values of distance and velocity for the ghost targets will be different. As for the real targets, the same values of distance and velocity can be obtained in the two groups. Therefore, as long as we find out coincident values from the two group values, the accurate distance and velocity of real targets can be obtained. A summary of this algorithm is shown in Fig. 3 .

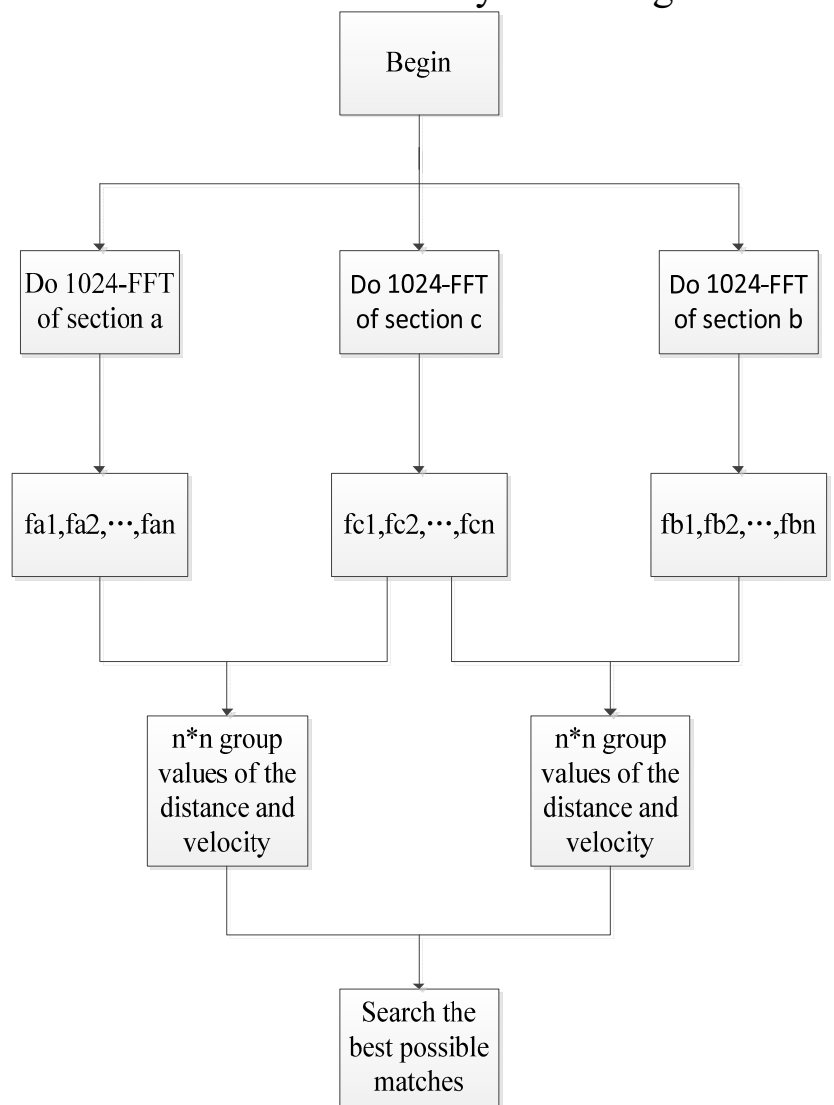

Fig. 3 Algorithm implementation process

\section{Results and Discussion}

The proposed algorithm was simulated in Matlab and the simulation parameters are listed in Table 1, while the targets parameters are listed in Table 2.

Table 1 Simulation Parameters

\begin{tabular}{ccc}
\hline Parameters & Values & Units \\
\hline fo & 24 & $\mathrm{GHz}$ \\
$\mathrm{B} 1$ & 200 & $\mathrm{MHz}$ \\
$\mathrm{B} 2$ & 50 & $\mathrm{MHz}$ \\
$\mathrm{T}$ & 5 & $\mathrm{~ms}$ \\
\hline
\end{tabular}

Table 2 Targets Parameters

\begin{tabular}{ccc}
\hline Target & Distance $(\mathrm{m})$ & Vlocity $(\mathrm{m} / \mathrm{s})$ \\
\hline 1 & 10 & 10 \\
2 & 20 & -15 \\
3 & 45 & 25 \\
4 & 70 & -35 \\
5 & 85 & -10 \\
\hline
\end{tabular}

Fig. 4 shows the simulation results of multi-targets detection using the proposed algorithm. The horizontal axis is the range and the vertical pole is the velocity. We can see that the real targets can be found and the ghost targets were canceled effectively. In addition, the benefit of the proposed scheme is that it can find out the real targets quickly with only slight computational complexity (three sections) rather than huge computational complexity. 


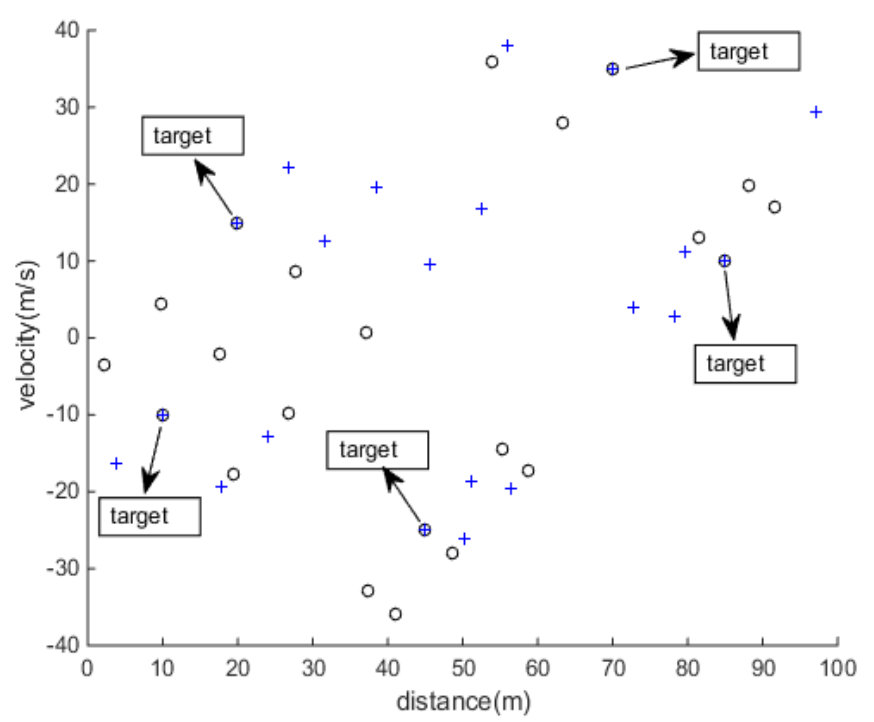

Fig4. The simulation results

\section{Conclusion}

A novel waveform and multi-target detection algorithm applied to FMCW automotive radar is proposed in this paper. The simulation results show that the real targets can be obtained accurately and this method cancels all the ghost targets simultaneously.

The improved method takes the features of simpler algorithm, less computation and higher efficiency, and is convenient for engineering realization, which indicate a possible potential application in FMCW radar technology.

\section{Acknowledgment}

This work is supported by the 2012 Distinguished Young Scientist awarded by the National Natural Science Foundation Committee of China (61225001), and by National Natural Science Foundation of China (6140010914, 61571231).

\section{References}

[1] Stove, A. G, Linear FMCW radar techniques, IEE Proceedings-F, Vol. 139, No. 5, Oct. 1992.

[2] H. Rohling, M. -M. Meinecke, Waveform design principles for automotive radar systems Radar, 2001 CIE International Conference on Proceedings,2001,pp.1-4.

[3] Amir Mukhtar, Likun Xia, and Tong Boon Tang, Vehicle Detection Techniques for Collision Avoidance Systems: A Review. IEEE Transactions on Intelligent Transportation Systems, vol, 16, 2015.

[4] Rohling, H., Moller, C, Radar waveform for automotive radar systems and applications, Radar Conference 2008, pp. 1-4, May 2008.

[5] Eugin Hyun, Woojin Oh, Jong-Hun Lee, Two-step Moving Target Detection Algorithm for Automotive 77GHz FMCW Radar, 2010 IEEE 72nd VTC Fall, Sept, 2010.

[6] Shunji Miyahara, New Algorithm for Multiple Object Detection in FM-CW Radar, SAE Technical paper series, 2004-01-0177, March 2004. 